\title{
Educação permanente para profissionais da área da saúde como estratégia de combate ao enfretamento da pandemia de COVID-19 na região norte: relato de experiência
}

\author{
Permanent education for health professionals as a strategy to combat the pandemic of \\ COVID-19 in the northern region: experience report \\ La educación permanente para los profesionales de la salud como estrategia para \\ combatir la pandemia de COVID-19 en la región norte: informe de experiência
}

Karina Negrão Zingra ${ }^{1 *}$, Anitha de Cássia Ribeiro da Silva², Ana Julia de Medeiros Fernandes², Arlindo Gonzaga Branco Junior ${ }^{2,3}$, Maxwendell Gomes Batista1.

\section{RESUMO}

Objetivo: Descrever o processo de capacitação à profissionais da saúde como forma de estratégia de combate ao enfrentamento da pandemia de covid-19 em municípios do Estado de Rondônia e do Amazonas. Relato de experiência: Foi realizado entre os meses de fevereiro e agosto de 2020 capacitações à profissionais de saúde por meio do processo de educação permanente. Se valendo de metodologias ativas como case based learning e ensino baseado em simulação. Foi proposto discussões de casos clínicos vivenciado no cotidiano por meio da formação de equipes multiprofissional e estimulação do debate e criação de estratégias para resolução afim de preparar melhor a atuação em equipe e desenvolver competências e habilidades em tais profissionais. Além disso, o uso de manequins realísticos possibilitou a aplicação prática como mais um aliado no processo de qualificação para futuros atendimentos e poder analisar a integração e estimular boas relações, preparando para um eficaz atendimento e resolutividade para os casos. Considerações finais: Tal processo, pode ser um facilitador para melhorar a estratégia de cuidado e integrar de forma harmônica as equipes que estariam na linha de frente ao combate do covid-19, lembrando-se que a integração e bons resultados no treinamento representariam melhor oferta e estratégia do cuidado.

Palavras-chave: Infecções por coronavirus, Educação permanente, Educação interprofissional.

\begin{abstract}
Objective: To describe the process of training health professionals as a form of strategy to combat the pandemic of the covid-19 in municipalities in the state of Rondônia and Amazonas. Experience report: Between February and August 2020, training for health professionals was carried out through the permanent education process. Using active methodologies such as case based learning and simulation-based teaching. Discussions of clinical cases experienced in daily life through the formation of multiprofessional teams and the stimulation of debate and the creation of strategies for resolution were proposed in order to better prepare team work and develop competencies and skills in such professionals. In addition, the use of realistic mannequins has enabled practical application as yet another ally in the qualification process for future care and to be able to analyze integration and encourage good relations, preparing for an effective service and resolving cases. Final considerations: Such a process can be a facilitator to improve the care strategy and harmoniously integrate the teams that would be in the front line to fight the covid-19, remembering that the integration and good training results would represent a better offer and care strategy.
\end{abstract}

Keywords: Coronavirus infections, Permanent education, Interprofessional education.

\footnotetext{
${ }^{1}$ Hospital Campanha de Rondônia, Porto Velho - RO. *E-mail: karina.zingra@gmail.com

2 Centro Universitário São Lucas, Porto Velho - RO

${ }_{3}^{3}$ Universidade Federal de Rondônia, Porto Velho - RO
} 


\section{RESUMEN}

Objetivo: Describir el proceso de formación de profesionales de la salud como una forma de estrategia para combatir la pandemia del covid-19 en los municipios del estado de Rondônia y Amazonas. Informe de experiencia: Entre febrero y agosto de 2020, se llevó a cabo la capacitación de profesionales de la salud a través del proceso de educación permanente. Utilizar metodologías activas como el aprendizaje basado en casos y la enseñanza basada en simulación. Se propuso la discusión de casos clínicos vividos en la vida cotidiana a través de la formación de equipos multiprofesionales y la estimulación del debate y la creación de estrategias de resolución con el fin de preparar mejor el trabajo en equipo y desarrollar competencias y habilidades en dichos profesionales. Además, el uso de maniquíes realistas ha permitido su aplicación práctica como un aliado más en el proceso de calificación para futuros cuidados y para poder analizar la integración y favorecer las buenas relaciones, preparar un servicio eficaz y resolver casos. Consideraciones finales: Tal proceso puede ser un facilitador para mejorar la estrategia de atención e integrar armoniosamente a los equipos que estarían en primera línea para combatir el covid-19, recordando que la integración y buenos resultados de capacitación representarían una mejor oferta y estrategia de atención.

Palabras clave: Infecciones por coronavirus, Educación permanente, Educación interprofesional.

\section{INTRODUÇÃO}

Desde o surto descrito de uma doença na China, a nova cepa de coronavírus, denominada SARS-CoV-2, causadora do COVID-19, ocasionou alarmante preocupação quanto ao aumento exponencial da demanda por serviços médico-hospitalares tanto na Ásia e Europa em poucas semanas (BRASIL, 2020).

Para atenuar tal cenário adotou-se medidas de suspenção de algumas atividades, aglomerações de pessoas como atividades escolares e do ensino superior, bares, comércio, empresas, eventos sociais e esportivos, shopping centers e quaisquer atividades de cunho social que necessita do contato entre pessoas (SILVA ACR, et al., 2020).

O COVID-19 em pouco tempo evoluiu de um surto para pandemia, em 11 de março de 2020. Tal fato, suscitou mudanças na organização de atendimento do Sistema Único de Saúde brasileiro e seus servidores, já sobrecarregados pela demanda de atendimento rotineiro, se depararam com um novo cenário e necessidade da oferta de atenção à saúde na urgência, emergência e cuidados intensivos resultante da taxa de complicações causadas pelo COVID-19 (BRASIL, 2020).

Essa doença se caracteriza por ser mais transmissível e ter um potencial de letalidade 14 vezes maior que a influenza, além de causar um contágio em progressão geométrica, tendo iniciado a transmissão comunitária no Brasil em 20 de março de 2020 e mais de 147 mil casos e 10 mil mortes confirmados até 9 de maio de 2020 (DAUMAS RP et al., 2020).

Com base nisso, o processo de capacitação profissional se faz necessário, uma vez que a educação permanente permite o encontro entre a formação e o trabalho, sendo relevante ao passo que qualifica, promove diálogos e reflexões sobre a atividade laboral a fim de transformar as práticas de saúde, bem como a assistência a população, permitindo maior efetividade e resolutividade na oferta de cuidado, estando alinhada à realidade de cada trabalhador (ALMEIDA WNM, et al., 2020; SILVA CPG, et al., 2020; MAROJA MCS, et al., 2020).

Os métodos de ensino aplicado nessas atividades possuem relevância para sua execução, diante disso, o case based learning, uma metodologia ativa, que permite a prática educativa pautada na aplicação de um caso clínico com o intuito de resgatar o conhecimento teórico prévio, montando uma ponte entre teoria e prática, contribuindo para motivação, reflexão crítica e integração dos saberes de todo o grupo presente foi implantada (ZINGRA KN, et al., 2020).

Com o intuito de complementar tais discussões, o ensino baseado em simulação (EBS) foi incluído no processo de capacitação e permite o reconhecimento preciso de falhas técnicas, reprodução de situações 
reais, treinar habilidades e repetir a execução de atividades básicas, sistematizado em um ambiente protegido, controlado e seguro garantindo o desenvolvimento da equipe para uma oferta de cuidado adequado (FORTE MI, et al., 2019).

O emprego dessas atividades foi ofertado a equipes multiprofissional, sendo relevante o processo educativo e prático interprofissional devido a integração entre equipes aumentar a resolutividade dos serviços, ter qualidade de atenção à saúde, evitar omissões ou duplicidade de cuidado, além de melhorar a comunicação entre profissionais, realidade necessária para as equipes que atuam na urgência, emergência e cuidados intensivos (PEDUZZI M, et al., 2012).

Diante disso, esse trabalho visa descrever o processo de capacitação por meio da aplicação de educação permanente para equipes multiprofisisonais, através da metodologia ativa e ensino baseado em simulação com abordagem de temas no contexto de urgência e emergência a fim de sistematizar o atendimento de pacientes durante a pandemia de Sars-cov- 2 .

\section{RELATO DE EXPERIÊNCIA}

Trata-se de um relato de experiência, no qual serão descritas capacitações para profissionais de saúde em meio a pandemia do COVID-19, no estado de Rondônia durante o período de fevereiro a agosto de 2020 utilizando a metodologia ativa case based learning e ensino baseado em simulação

A metodologia ativa escolhida para a palestra foi o case based learning e envolveu resolução de casos clínicos utilizando problemáticas vivenciadas no cotidiano de cada local. Já quando envolvia simulações os profissionais eram inseridos em ambiente realístico de atendimento ao paciente. $O$ objetivo das palestras era sistematizar e padronizar o atendimento aos pacientes com quadro clínico de COVID 19 a fim de garantir segurança dos profissionais.

Case based learning consiste em metodologia de ensino facilmente aplicado, desde que haja um caso estruturado para analisar. Sendo assim, há necessidade de conhecimento adquirido pelos ouvintes previamente. A partir de um caso clínico a teoria é resgatada e ministrada aplicada no caso apresentado. $O$ instrutor guia e desenvolve a capacidade de raciocínio dos participantes conectando teoria com atividades práticas. (MONTANHER VC, 2012)

O ensino baseado em simulação, permite que os participantes se deparem com uma problemática, criem hipóteses baseadas nos dados clínicos do caso proposto. Desta maneira, apoiam-se e conhecimentos prévios para que cheguem a conclusão de qual conduta mais adequada para adotarem naquele momento. Esta forma de aprendizagem encontra necessidades de formar profissional capaz de raciocínio ao analisar problemas e tomar decisões fundamentadas em seus conhecimentos (DOURADO ASS e GIANELLA TR, 2014).

As duas metodologias apresentadas foram escolhidas como metodologias alternativas os habituais utilizados nas academias a fim de proporcionar melhor dinâmica e aprendizagem entre os participantes. Como os profissionais escolhidos em sua maioria são antigos em seus ambientes de trabalho, poderia ser encontrada maior resistência na participação de atividade expositiva que envolvesse pouca participação.

O público alvo da capacitação foram enfermeiros, técnicos de enfermagem, fisioterapeutas, fonoaudiólogos e médicos sendo enviado convite via grupos de comunicação digital. As cidades envolvidas no projeto presencial foram nos Estados de Rondônia e Amazonas. Em outro momento, foi realizada a capacitação via plataforma digital para maior alcance do público alvo em outros estados do Brasil. Participaram, das atividades proporcionadas, um total de 563 profissionais da saúde.

Os principais temas abordados na capacitação envolveram reanimação cardiopulmonar, intubação orotraqueal e outras vias aéreas avançadas, manobra de pronação de pacientes críticos, paramentação, higienização das mãos e cuidados com matérias de proteção individual. As palestras foram realizadas entre fevereiro e agosto de 2020, durante a rotina de cada instituição de saúde, promovida por um professor graduado em medicina e instrutor do curso de Advanced Cardiovascular Life Suport 2015 (ACLS). A oficina era oferecida em período diurno ou noturno, durante o plantão da equipe de cada instituição conforme horário que houvesse melhor adesão do público alvo. 
Em um primeiro momento, os funcionários se dividiram no plantão para que uma parte assistisse a palestra, enquanto, o atendimento na unidade de saúde continuasse normalmente. $O$ treinamento era realizado em local que tivesse maca, rede de oxigênio e ar comprimido, lençóis, monitor multiparamétrico, desfibrilador, dispositivo bolsa válvula-máscara, cateter nasal, mascara não reinalante, entre outros materiais que seriam utilizados para o atendimento de paciente potencialmente graves.

Foi necessário também uso de boneco de treinamento para reanimação cardiopulmonar. As manobras de pronação eram treinadas chamando um dos ouvintes como voluntário para maior realidade na movimentação para realização de tal manobra. Após a organização do setor, a palestra começava com o instrutor apresentando um caso clínico e questionava aos funcionários como eles realizariam o atendimento simulado daquele paciente. Cerca de 30 minutos de discussão, a palestra era direcionada para corrigir os principais equívocos que a equipe havia cometido.

A simulação consistia em colocar os profissionais em simulação realística de atendimento inicial ao paciente grave. $O$ instrutor simulava verbalmente a clínica e queixas do paciente (boneco de reanimação posicionado em decúbito ventral na maca), enquanto os profissionais de saúde realizavam o atendimento desde a monitorização, condutas e manobras de reanimação cardiopulmonar. Entretanto, nas palestras expositivas, os profissionais recebiam um caso clínico escrito e debatiam as condutas e medidas que poderiam ser realizadas para estabilização do paciente.

Ao finalizar a simulação e correções com o instrutor, eram abordados os temas de forma intercalada entre apresentação expositiva do caso clínico e treinamento simulado entre os funcionários. Sendo que, em grande maioria, os grupos que participavam das simulações concordaram terem se sentido mais inseridos e gerado maior aprendizado em relação aos grupos que participaram da atividade mais expositiva. Tanto instrutor como alunos concordaram que as duas metodologias proporcionavam aprendizado por: utilizar o erro como chance de aprender; homogeneizou o trabalho em equipe; relacionou o mundo teórico com a prática clínica.

A oportunidade do feedback imediato foi ressaltada diversas vezes durante o treinamento da equipe. Isso por proporcionar retorno em tempo hábil correção do erro, retirada de dúvidas pertinentes e discussão de condutas que costumavam ser divergentes entre os profissionais. $O$ aproveitamento do erro como oportunidade de aprender, mostrou como a visualização, manipulação e interpretação de situações complexas é um enorme potencial a ser aproveitado em metodologias de ensino como o case based learning e ensino baseado em simulação (DOURADO ASS e GIANELLA TR, 2014).

\section{DISCUSSÃO}

A partir do processo de disseminação do sars-cov-2 se apresentar maior que outros vírus de mesma espécie, foi descrito que cada doente pode infectar até duas a três pessoas (GALLASCH CH, et al, 2020). Diante disso, o alastramento sustentado para dois ou mais continentes decretou o COVID 19 como uma pandemia incentivando alterações nas dinâmicas de demanda e oferta de atendimento (MARQUES LC, et al., 2020).

Conforme esse cenário, a expansão de assistência de forma planejada e organizada, relacionando os fatores necessários para o enfretamento da crise, como: recursos, profissionais de saúde, espaço e comunicação eficaz, tornou-se necessário como estratégia de combate (BRANCO A, et al, 2020). Assim, o desenvolvimento de capacitação se tornou um pilar importante, ao passo que, envolve diversos atores permitindo a aplicação da educação interprofissional, intercâmbio de saberes entre grupos de diversas áreas, estimulando a colaboração e melhorando resultado na saúde (SOUZA SV e ROSSIT RAS, et al., 2020).

A educação interprofissional tem sido objeto de estudo em diversos países desenvolvidos devido a premissa que um ou mais profissionais de áreas distintas aprendem e aplicam melhor o trabalho em conjunto o que interfere diretamente na melhoria da qualidade de assistência a população (ROSSIT RAS, et al., 2018). Diante disso, integrar as equipes e prepara-las para o enfrentamento da pandemia que apresenta uma taxa de $15 \%$ dos acometidos necessitando de oxigenoterapia e 5\% de internação em unidade de terapia intensiva (UTI) é de grande valia para mitigar os números de mortes decorrente da COVID-19 (NORONHA KVMS, et al. 2020). 
A estratégia de ensino, como o case based learning, escolhido ao propor as problemáticas vivenciadas no cotidiano e promover o debate em grupo pode ser um fator chave para estimular boas relações entre as equipes, já que, no atendimento de urgência e emergência necessita da integração multiprofissional e uma má comunicação e relação pode acarretar em atrasos no diagnóstico e tratamento (INDRUCZAKI NS, et al., 2020).

A metodologia ativa, exemplificada pelo case based learming se torna mais efetiva no seu processo de aprendizado quando comparada a tradicional, ao passo que supera o modelo da didática técnica, baseada apenas na transmissão de conteúdo, mesmo se valendo de tecnologias, não deixando o professor como ator principal responsável pelo processo de ensino aprendizagem e também alterando a ordenação física da sala, estimulando a superação do processo de disciplina e autoridade desenvolvida no modelo tradicional (RODRIGUES LP, et al., 2011).

Ressalta-se ainda a possibilidade que a metodologia ativa proporciona ao envolver os alunos nas tomadas de decisão referente ao processo de aprendizagem, introduzindo casos reais, incentivando o desenvolvimento de habilidades que não seriam possíveis se a estrutura se mantivesse com foco no modelo tradicional unidirecional fosse a escolha inicial para desenvolvimento de raciocínio clínico e crítico, e integração em equipe tão fundamental nesse momento (NOBRE JCS, et al., 2006).

Somado a isso, o emprego do ensino baseado em simulação permitiu a aplicação prática e melhor desenvolvimento das habilidades necessárias aos profissionais que iriam atuar na linha de frente, sendo de suma importância, pois profissionais que atuam na área da medicina, enfermagem ou técnicos de enfermagem relatam que há déficit no ensino da urgência e emergência durante a formação profissional na academia (OLIVEIRA OS, et al., 2020). Tal fato, pode ser um fator que contribui para insegurança de integrantes da equipe, tomada de decisão errada, aumento das tensões e cobranças na tentativa de fornecer um atendimento de qualidade, efetivo e resolutivo daqueles pacientes que chegam para ser atendidos.

Esse modelo possibilita superar o aprendizado por meio de técnicas passivas que foi predominante durante a graduação, por meio do ensino baseado em simulação há um aprendizado consistente e atenuando possíveis desfechos desfavoráveis durante a abordagem médica e da equipe, promovendo a retenção de conhecimento por um espaço de tempo mais prolongado tornando o aprendizado mais dinâmico e prazeroso quando comparado ao estilo passivo de ensino (FLATO UAPF, et al., 2011).

Contornar a problemática da formação deficiente, má comunicação entre equipe e melhorar a tomada de decisão se faz imprescindível devido à mudança na rotina do serviço de saúde com o aumento de internações hospitalares por complicações respiratórias, choque séptico ou falência múltipla de órgãos relacionada ao COVID-19 (GALLASCH CH, et al, 2020). Ademais, os profissionais que atuam ainda têm que lidar com a superlotação das unidades de saúde, falta de leitos e equipamentos, fato que intensifica as tensões nas equipes e podem suscitar um ambiente pouco harmônico (MARQUES LC, et al., 2020).

Vale ressaltar que o trabalho em equipe aconteceu durante todo o período de treinamento em ambas as metodologias, sendo mais executado quando utilizada a metodologia de ensino baseado em simulação. Assim, além de conhecimentos de habilidades específicas (intubar, manobras de compressão torácica, desfibrilar, ventilar, administrar medicamentos) os profissionais tiveram oportunidade de treinar o papel de liderar, ser responsável por condutas e integrar membros da equipe a fim de resolver o caso proposto (DOURADO ASS e GIANELLA TR, 2014).

Preparar o arsenal laboral para enfrentar a nova problemática de saúde pública também se valeu da dinâmica feita por meio de plataformas digitais e ensino a distância, fato que já ocorreu como nos processos de capacitação para combate do vírus da Influenza (SILVA ASR, et al., 2019). O uso da rede mundial de computadores permite uma atualização efetiva, mais acessível e integra profissionais de diversos lugares potencializando o processo de construção de conhecimento, debate e problematização da temática em questão permitindo uma rápida difusão de informação (OBREGÓN PL, et al., 2019).

Desse modo, usar das funcionalidades que a internet permite, associar as equipes que atuam de forma conjunta e propor um ensino ativo com a aplicação prática é importante ao romper o aprendizado tradicional o tornando mais fluido, flexível e atraente potencializando o desenvolvimento de cada profissional presente 
(ZUCATTI APN, et al., 2018). Assim, o preparo de profissionais em diversas regiões do Estado possibilitou contornar possíveis danos ao cuidado ofertado durante a nova pandemia (MARQUES LC, et al., 2020).

Diante do exposto, este relato teve como objetivo demonstrar a aplicação do método case based learning na capacitação de profissionais de saúde durante a pandemia do SARS-COVID 19. Os profissionais demostraram boa adesão a metodologia devido fácil aplicabilidade prática no contexto do serviço no qual estão inseridos. A inclusão de metodologias ativas pode ampliar a visão dos profissionais e devem ser utilizadas em capacitações de outros momentos a fim de desenvolver habilidades que respondam às suas necessidades e consolidar o ensino continuado.

\section{REFERÊNCIAS}

1. ALMEIDA WNM, et al. Educação permanente como ferramenta de integração entre agentes de saúde e de endemias. Promoção da Saúde, 2020; 33: 1-7.

2. BRANCO A. Serviço de emergência hospitalar: fluxos de atendimento a pacientes suspeitos ou confirmados para covid-19. Enfermagem em Foco, 2020; 11(1): 199-204.

3. BRASIL. Ministério da saúde. (org.). Protocolo de manejo clínico do coronavírus (covid-19) na atenção primária à saúde. 2020.

4. BRASIL. UNA SUS. (org.). Organização Mundial de Saúde declara pandemia do novo Coronavírus: mudança de classificação obriga países a tomarem atitudes preventivas. 2020.

5. BRASIL. Ministério da Saúde. Secretaria de Atenção Especializada À Saúde (org.). Protocolo de Tratamento do Novo Coronavírus (2019-nCoV). Disponível em: https://www.arca.fiocruz.br/bitstream/icict/40195/2/Protocolo_Tratamento_Covid19.pdf. Acesso em: 27 jul. 2020.

6. COSTA MV, et al. Aspectos institucionais para a adoção da Ēducação Interprofissional na formação em enfermagem e medicina. Saúde em Debate, 2019; 43(1), 64-76.

7. DAUMAS RP, et al. O papel da atenção primária na rede de atenção à saúde no Brasil: limites e possibilidades no enfrentamento da COVID-19. Caderno de Saúde Pública, 2020; 6(36): 1-7.

8. DOURADO ASS, GIANELLA TR. Ensino Baseado em Simulação na Formação Continuada de médicos: análise das Percepções de alunos e Professores de um Hospital do rio de Janeiro. Revista Brasileira de educação médica. 2014; 38(4): 460-469.

9. FLATO UAP, et al. Educação baseada em simulação em medicina de urgência e emergência: a arte imita a vida. Rev Bras Clin Med, 2011; 5(9): 360-364.

10. FORTE MI, et al. Evolução da autoconfiança e segurança de estudantes após aprendizagem utilizando manequins em Periodontia. Revista Abeno, 2019; 19(1): 106-114.

11. FREIRE FR, et al. Educação Interprofissional nas políticas de reorientação da formação profissional em saúde no Brasil. Saúde em Debate, 2019; 43(1): 86-96.

12. GALLASCH CH, et al. Prevenção relacionada à exposição ocupacional do profissional de saúde no cenário de COVID-19. Revista Enfermagem Uerj, 2020, 28: 1-6.

13. INDRUCZAKI NS, et al. Conflitos entre as equipes de saúde na transferência do cuidado pré-hospitalar. Revista Enfermagem Uerj, 2020; 28: 1-7.

14. MAROJA MCS, et al. Os desafios da formação problematizadora para profissionais de saúde em um programa de residência multiprofissional. Interface (Botucatu). 2020; 24: e180616.

15. MARQUES LC, et al. Covid-19: cuidados de enfermagem para segurança no atendimento de serviço pré-hospitalar móvel. Texto Contexto Enferm [Internet]. 2020; 29:e20200119.

16. MONTANHER VC. Aprendizagem baseada em casos nas aulas de física do ensino médio, Universidade Estatual de Campinas, Faculdade de Educação, Campinas, São Paulo, 2012.

17. NOBRE JCS, et al. Prendizagem Baseada em Projeto (Project-BasedLearning - PBL) aplicada a software embarcado e de temporeal. In: XVII simpósio brasileiro de informática na educação, 2006; 1: 1-10.

18. NORONHA KVMS, et al. Pandemia por COVID-19 no Brasil: análise da demanda e da oferta de leitos hospitalares e equipamentos de ventilação assistida segundo diferentes cenários. Caderno de Saúde Pública, 2020; 6(36): 1-17.

19. OBREGÓN PL, et al. Utilização de recursos de tele-educação em um hospital universitário da região oeste do Paraná. Saúde em Rede, 2019; 5(2), 89-102.

20. OLIVEIRA OS, et al. Professional performance in urgencies/emergencies on basic units of health. Revista de Pesquisa Cuidado É Fundamental Online, 2020; 820-826

21. ROSSIT RAS, et al. Construção da identidade profissional na Educação Interprofissional em Saúde: percepção de egressos. Interface: Comunicação Saúde, 2018: 22(1): 1-12.

22. RODRIGUES LP, et al. O tradiocional e o moderno quanto à didática no ensino superior. Revista Científica do Itpac, 2011; 4(3): 1-9.

23. SILVA TPS, et al. Tele-education applied to human communication health to cope with triple epidemics in the state of Pernambuco, Brazil: an experience report. Revista Cefac, 2020: 22(3): 10-19.

24. SILVA ASR. Construção de materiais educativos digitais para uma capacitação online sobre influenza: relato de experiência. Saúde em Rede, 2019; 5(3): 227-239.

25. SILVA CPG, et al. Atividades educativas para uso adequado de equipamentos de proteção individual em hospital federal de referência. Enfermagem Foco, 2020; 1: 228-233. 
26. SILVA ACR, SILVA TM. Aplicação do Arco de Maguerez no planejamento de aulas teóricas remotas devido às alterações sociais frente a pandemia covid-19: relato de experiência. A sociedade em tempos de covid-19. Editora inovar 2020: 421 - 429.

27. SOUZA SV, ROSSIT RAS. dilemas e perspectivas dos recursos humanos em saúde no contexto da pandemia. Enfermagem em Foco, 2020; 11(1): 68-73.

28. PEDUZZI M. Educação Interprofissional: Formação De Profissionais De Saúde Para O Trabalho Em Equipe Com Foco Nos Usuários. Rev Esc Enferm Usp, 2012; 47(4): 977-983.

29. ZINGRA KN, et al. Utilização do método case based learning em uma oficina de educação interprofissional para profissionais docentes da área da saúde: relato de experiência. Diálogos: Economia e Sociedade, 2020; 1: 18-24.

30. ZUCATTI APN, et al. Criação de uma Simulação para o Desenvolvimento de Competências em um Hospital. Psicologia Ciência e Profissão, 2018; 39: 1-15. 